\title{
Präparative und spektroskopische Untersuchungen zur Dimorphie von $\mathrm{CsNiF}_{3}$
}

\author{
Preparative and Spectroscopic Investigations on the Dimorphy of $\mathrm{CsNiF}_{3}$
}

\author{
R. Haegele, D. Babel und D. Reinen \\ Sonderforschungsbereich 127 (Kristallstruktur und chemische Bindung) und Fachbereich Chemie \\ der Universität Marburg
}

(Z. Naturforsch. 31 b, 60-65 [1976]; eingegangen am 27. August 1975)

Fluoro-perovskite, Dimorphism, Ligand Field Spectra

\begin{abstract}
While the stable modification of $\mathrm{CsNiF}_{3}$ is of green colour, a yellow phase is obtained by fluorination of $\mathrm{CsNiCl}_{3}$ and decomposition of the product " $\mathrm{CsNiF}_{4}$ ", which contains $\mathrm{Ni}^{4+}$ and $\mathrm{Ni}^{3+}$ besides $\mathrm{Ni}^{2+}$. The two modifications have the $2 \mathrm{~L}$ - and $9 \mathrm{~L}$-perovskite-structure, respectively, and are characterized by their X-ray patterns and low-temperature ligand field spectra. The differences in colours and spectral band positions are discussed with respect to the $\mathrm{Ni}-\mathrm{F}-\mathrm{Ni}$ bond angles.
\end{abstract}

\section{Einleitung}

Die Verbindung $\mathrm{CsNiF}_{3}$ ist früher von uns als gelbgrüne Substanz beschrieben worden ${ }^{1}$, die in der hexagonalen 2L-Perowskitstruktur der Verbindungen $\mathrm{CsNiCl}_{3}{ }^{2}$ und $\mathrm{BaNiO}_{3}{ }^{3}$ kristallisiert ${ }^{1,4}$ (Abb. 1).

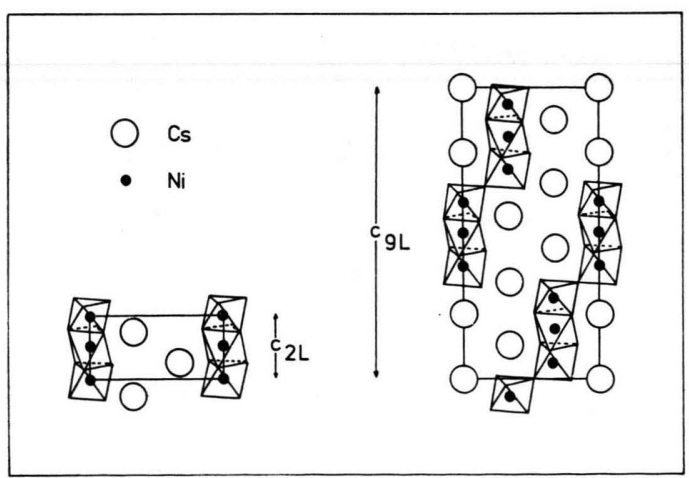

Abb. 1. (110)-Schnitte der hexagonalen Perowskitstrukturen vom $\mathrm{BaNiO}_{3}(2 \mathrm{~L})^{3}$. und $\mathrm{BaRuO}_{3}(9 \mathrm{~L})^{8}$ Typ $(2 \mathrm{~L}=2,9 \mathrm{~L}=9$ Lagen dichtest gepackter Schichten innerhalb der Identitätsperiode c).

Unter den genannten isostrukturellen Perowskitvarianten, die Kettenstrukturen mit nur über Flächen verknüpften Oktaedern darstellen, ist die Fluorverbindung wegen ihres 1-dimensional ferro-

Sonderdruckanforderungen an Dr. D. BABEL, Fachbereich Chemie der Philipps-Universität, D-3550 Marburg/Lahn, Lahnberge, BRD. magnetischen Charakters besonders interessant ${ }^{5}$. Um für Neutronenbeugungsuntersuchungen ${ }^{5,6}$ geeignete Einkristalle von $\mathrm{CsNiF}_{3}$ zu züchten, hatten wir größere Substanzmengen dieser Verbindung darzustellen. Dabei beobachteten wir von den Darstellungsbedingungen abhängige Farbunterschiede zwischen oder Inhomogenitäten in den aus $\mathrm{CsHF}_{2}$ und $\mathrm{NiF}_{2}$ erhaltenen Sinterproben, die häufig einerseits grüne, andererseits gelbe Bestandteile erkennen ließen. Die nähere Untersuchung ergab, daß hierfür die Existenz einer zweiten, rein gelben $\mathrm{CsNiF}_{3}$ Modifikation mit verantwortlich zu machen war.

\section{Präparative \\ und röntgenographische Untersuchungen}

Leuchtend hellgrünes $\mathrm{CsNiF}_{3}$ mit der erwähnten hexagonalen $2 \mathrm{~L}$-Struktur bildet sich bei längerem Erhitzen einer Mischung von $\mathrm{CsHF}_{2}+\mathrm{NiF}_{2}$ unter Inertgas in Platinröhrchen auf mindestens $800{ }^{\circ} \mathrm{C} 1$. Wenn man nur wenige Stunden und/oder nicht so hoch erhitzt, ist das Sinterprodukt gelb und mehrphasig: Die Röntgenpulverdiagramme lassen dann zwar meist neben unumgesetztem $\mathrm{NiF}_{2}$ und der cäsiumreicheren orthorhombischen Verbindung $\mathrm{Cs}_{4} \mathrm{Ni}_{3} \mathrm{~F}_{10}{ }^{7}$ auch $2 \mathrm{~L}-\mathrm{CsNiF}_{3}$ erkennen, weisen aber noch zusätzliche Interferenzen auf, deren Zahl und Intensität stark von den Darstellungsbedingungen der Proben abhängt. Dabei trat jedoch wiederholt ein Beugungsmuster hervor, das uns vom Pulver- 
diagramm der hexagonal-rhomboedrisch in einer 9L-Perowskitvariante ${ }^{8}$ kristallisierenden Verbindung $\mathrm{CsCoF}_{3} 1$ bekannt war (Abb. 1). Mit den Gitterkonstanten $a=6,14_{9}, c=22,35 \AA$ (s. Tab. I) gelang die Indizierung der entsprechenden Interferenzen, die auf die Existenz einer zweiten $\mathrm{CsNiF}_{3}$ Modifikation mit 9L-Struktur hinwiesen. Unter geeigneten Bedingungen dargestellte und dann stets farblich inhomogene Sinterproben mit grünen und gelben Zonen zeigten röntgenographisch nur die Reflexe der beiden Phasen mit 2L- bzw. 9L-Struktur in wechselnden Proportionen. Im Gegensatz zur Darstellung der reinen, grünen 2L-Form gelang es jedoch in diesen Temperversuchen nicht, die offensichtlich bei tieferen Temperaturen bevorzugte gelbe 9L-Form von $\mathrm{CsNiF}_{3}$ rein darzustellen.

Aus diesem Grunde wurde nach anderen Darstellungsweisen für 9L-CsNiF 3 gesucht. Schon bekannt war die Hochdrucksynthese $(5 \mathrm{kbar})$ dieser Modifikation, für die Longo und KafaLas ${ }^{9,10}$ in guter Ubereinstimmung mit unseren Werten Gitterkonstanten von $a=6,15, c=22,32 \AA$ angaben. Ein einfacherer Weg zur Darstellung größerer Mengen der 9L-Form ergab sich durch Fluorierung von $\mathrm{CsNiCl}_{3}$ und thermischen Abbau des als Zwischenprodukt erhaltenen ,, $\mathrm{CsNiF}_{4}$ "11.

Die Verbindung $\mathrm{CsNiCl}_{3}$, leicht durch Fällung alkoholischer Lösungen von wasserfreiem $\mathrm{NiCl}_{2}$ mit $\mathrm{CsCl} \mathrm{zu}$ erhalten, setzt sich im Fluorwasserstoffstrom selbst bei $600{ }^{\circ} \mathrm{C}$ nur unvollständig zu $\mathrm{CsNiF}_{3}$ um. Dagegen führt die Reaktion mit elementarem Fluor bei $2-300{ }^{\circ} \mathrm{C}$ nach wenigen Stunden zu einem Produkt der analytisch überprüften Bruttozusammensetzung , , $\mathrm{CsNiF}_{4}$ “. Dabei handelt es sich jedoch um keine einheitliche Verbindung. Die röntgenographische Pulveruntersuchung ergab, daß sich nach

$$
\begin{aligned}
& 3 \mathrm{CsNiCl}_{3}+6 \mathrm{~F}_{2} \underset{-9 / 2 \mathrm{Cl}_{2}}{\longrightarrow} 3,{ }_{,} \mathrm{CsNiF}_{4} "= \\
& \mathrm{Cs}_{2} \mathrm{Ni}^{\mathrm{IV}} \mathrm{F}_{6}+\mathrm{CsNi}^{\mathrm{II}} \mathrm{Ni}^{\mathrm{III}} \mathrm{F}_{6}
\end{aligned}
$$

ein Gemisch der beiden kubischen Verbindungen $\mathrm{Cs}_{2} \mathrm{NiF}_{6}$ (weinrot, $a=8,94 \AA$ ) und $\mathrm{CsNi}_{2} \mathrm{~F}_{6}$ (braun, $a=10,13 \AA$ ) gebildet hatte ${ }^{11}$. Die Ni(IV)-Verbin- dung, mit derselben Gitterkonstante und Farbe bereits von BoDE und Voss ${ }^{12}$ beschrieben, kristallisiert im $\mathrm{K}_{2} \mathrm{PtCl}_{6}$-Typ. Die Verbindung $\mathrm{CsNi}^{\mathrm{II}} \mathrm{Ni}^{\mathrm{III}} \mathrm{F}_{6}$, bemerkenswert durch die gegen Fluorierung beständige gemischte Nickelvalenz, ist ein neuer Vertreter des $\mathrm{RbNiCrF}_{6}-\mathrm{Typs}^{13}$. Ihre Gitterkonstante liegt, in Ubereinstimmung mit dem besonders kleinen Ionenradius sowohl des 2- als auch des 3wertigen Nickels, noch unterhalb der für die isostrukturellen Verbindungen $\mathrm{CsNiCrF}_{6}(a=10,28 \AA)^{14}$ und $\mathrm{CsZnNiF}_{6}$ (braun, $\left.a=10,17 \AA\right)^{15}$ angegebenen Werte.

Die Präsenz von $\mathrm{Ni}^{3+}$-Ionen $\mathrm{im},{ }_{\text {,CsNiF}}{ }_{4}$ “ läßt sich ebenso spektroskopisch belegen. Das ESRSpektrum zeigt das für Low-Spin- $\mathrm{Ni}^{3+}$-Ionen in tetragonal geweiteter oktaedrischer Koordination charakteristische anisotrope Signal mit $\mathrm{g}_{||}=\mathbf{2 , 0 5}$ und $\mathrm{g}_{1}=2,35_{5}\left(130^{\circ} \mathrm{K}\right)^{16}$. Ebenso findet sich im Ligandenfeldspektrum der die Aufspaltung des ${ }^{2} \mathbf{E}_{\mathbf{g}}$ Zustandes angebende Übergang als langwelligste Bande bei $7500 \mathrm{~cm}^{-1}$.

Das genannte Fluorierungsprodukt von $\mathrm{CsNiCl}_{3}$ spaltete bei mehrstündigem Erhitzen auf $400-500^{\circ} \mathrm{C}$ unter strömendem Argon Fluor ab und hinterließ ein rein gelbes Pulver der Zusammensetzung $\mathrm{CsNiF}_{3}$. Die Röntgenpulveraufnahme ist in Abb. 2 in Form eines Strichdiagramms dem der grünen Modifikation

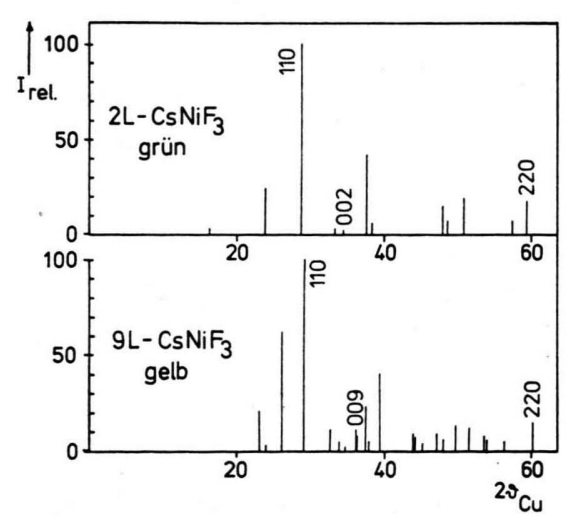

Abb. 2. Strichdiagramme von Röntgenpulveraufnahmen von $2 \mathrm{~L}$ - und $9 \mathrm{~L}-\mathrm{CsNiF}_{3}\left(\mathrm{CuK}_{\alpha}\right.$-Strahlung).

\begin{tabular}{|c|c|c|c|c|c|c|c|}
\hline & Farbe & $a[\AA]$ & $c[\AA]$ & $c / a$ & $z$ & $\mathrm{~V} / z\left[\AA^{3}\right]$ & Strukturtyp \\
\hline $\mathrm{CsNiF}_{3}$ & grün & $6,23_{6}$ & $5,22_{5}$ & 0,838 & 2 & 87,98 & $2 \mathrm{~L}, \mathrm{BaNiO}_{3}{ }^{3}$ \\
\hline $\mathrm{CsNiF}_{3}$ & gelb & $6,14_{9}$ & $22,3_{5}$ & $\frac{9}{2} \times 0,808$ & 9 & 81,32 & $9 \mathrm{~L}, \mathrm{BaRuO}_{3} 8$ \\
\hline
\end{tabular}

Tab. I. Hexagonale Gitterkonstanten der beiden $\mathrm{CsNiF}_{3}$-Modifikationen. 
gegenübergestellt. Mit den in Tab. I angegebenen Gitterkonstanten sind die beiden Aufnahmen jeweils vollständig zu indizieren. Für die gelbe 9LForm ist dies in Tab. II gezeigt.

Tab. II. Hexagonale Indizierung des rhomboedrischen $9 \mathrm{~L}-\mathrm{CsNiF}_{3}$.

\begin{tabular}{rllllr}
\hline $\mathrm{I}_{\text {rel }}$ & $\mathrm{d}_{\text {beob }}[\AA]$ & $\mathrm{d}_{\text {ber }}[\AA]$ & $h$ & $k$ & $l$ \\
\hline 21 & 3,8601 & 3,8549 & 1 & 0 & 4 \\
3 & 3,7231 & 3,7250 & 0 & 0 & 6 \\
62 & 3,4295 & 3,4237 & 0 & 1 & 5 \\
100 & 3,0810 & 3,0745 & 1 & 1 & 0 \\
11 & 2,7377 & 2,7384 & 1 & 0 & 7 \\
5 & 2,6457 & 2,6439 & 0 & 2 & 1 \\
2 & 2,5772 & 2,5899 & 2 & 0 & 2 \\
13 & 2,4833 & 2,4833 & 0 & 0 & 9 \\
8 & 2,4754 & 2,4740 & 0 & 1 & 8 \\
23 & 2,4044 & 2,4036 & 0 & 2 & 4 \\
5 & 2,3708 & 2,3712 & 1 & 1 & 6 \\
41 & 2,2880 & 2,2875 & 2 & 0 & 5 \\
9 & 2,0601 & 2,0608 & 1 & 0 & 10 \\
8 & 2,0446 & 2,0449 & 0 & 2 & 7 \\
4 & 2,0010 & 2,0045 & 2 & 1 & 1 \\
9 & 1,9275 & 1,9274 & 2 & 0 & 8 \\
6 & 1,8942 & 1,8936 & 2 & 1 & 4 \\
13 & 1,8351 & 1,8353 & 1 & 2 & 5 \\
13 & 1,7751 & 1,7751 & 3 & 0 & 0 \\
8 & 1,7092 & 1,7118 & 0 & 2 & 10 \\
6 & 1,7018 & 1,7027 & 2 & 1 & 7 \\
5 & 1,6327 & 1,6331 & 1 & 2 & 8 \\
15 & 1,5369 & 1,5373 & 2 & 2 & 0 \\
\hline
\end{tabular}

Aus den in Tab. I mit aufgeführten reduzierten Zellvolumina V/z ergibt sich für die als Tieftemperaturmodifikation aufzufassende 9L-Form ein um $7,6 \%$ kleinerer Wert, als für die 2L-Hochtemperaturform. Dies ist mit dem erwähnten Auftreten der 9L-Form als Hochdruckphase ${ }^{9,10}$ in Einklang. Die entsprechende Beobachtung, daß Tieftemperaturund Hochdruckform identisch sind, machten wir auch bei dem strukturverwandten Elpasolith $\mathrm{Cs}_{2} \mathrm{NaFeF}_{6}{ }^{11,17}$. Wie dort fanden wir eine quantitative Umwandlung der Tief- in die Hochform jedoch erst nach Erhitzen auf den Schmelzpunkt, der im Falle von $\mathrm{CsNiF}_{3}$ bei $870{ }^{\circ} \mathrm{C}$ liegt ${ }^{11}$.

Die Kristallstrukturen der beiden $\mathrm{CsNiF}_{3}$-Modifikationen unterscheiden sich durch die verschiedene Stapelfolge der $\mathrm{CsF}_{3}$-Schichten, zwischen denen die Nickelionen Oktaederlücken besetzen ${ }^{18}$. Im Gegensatz zur ausschließlichen Flächenverknüpfung der Oktaeder in der Kettenstruktur des 2L-Typs, kommen in der 9L-Struktur zusätzlich auch Eckenverknüpfungen vor, durch die Dreiergruppen flächenverknüpfter Oktaeder dreidimensional vernetzt sind (s. Abb. 1). Es sei an dieser Stelle erwähnt, daß analoge Dreiergruppen auch die Struktur der gleichfalls gelben Verbindung $\mathrm{Cs}_{4} \mathrm{Ni}_{3} \mathrm{~F}_{10}{ }^{7}$ charakterisieren. Möglicherweise übt die Anwesenheit geringer $\mathrm{Cs}_{4} \mathrm{Ni}_{3} \mathrm{~F}_{10}$-Mengen sogar einen stabilisierenden Einfluß auf die $9 \mathrm{~L}$-Form von $\mathrm{CsNiF}_{3}$ aus. Für diese Annahme spricht, daß sich bei den Sinterversuchen aus $\mathrm{CsHF}_{2}$ und $\mathrm{NiF}_{2}$ die $9 \mathrm{~L}-\mathrm{Phase}$ bevorzugt $\mathrm{zu}$ bilden schien, wo mit geringem lokalen CsF-Überschuß zu rechnen war: Einerseits in der Anfangsphase der Umsetzung (mit $\mathrm{Cs}_{4} \mathrm{Ni}_{3} \mathrm{~F}_{10}$ als Zwischenprodukt), andererseits bei längerem Erbitzen und dadurch ausgelöster Zersetzung $\mathrm{CsNiF}_{3} \rightarrow \mathrm{CsF}+\mathrm{NiF}_{2}$ mit CsF-Anreicherung in den kälteren Reaktionszonen. Möglicherweise spielen auch bei der Darstellung von $9 \mathrm{~L}-\mathrm{CsNiF}_{3}$ durch Thermolyse von „, $\mathrm{CsNiF}_{4}$ “ cäsiumreichere $\mathrm{Zwischenprodukte} \mathrm{eine}$ Rolle. Ein geringer, wasserunlöslicher $\mathrm{NiF}_{2}$-Rest, der bei vereinzelten Proben beobachtet wurde, weist in diese Richtung. Röntgenographisch traten jedoch keine Fremdreflexe in den Pulverdiagrammen in Erscheinung.

Die für die gelbe Verbindung $\mathrm{Cs}_{4} \mathrm{Ni}_{3} \mathrm{~F}_{10}$ gefundenen Ni-F-Abstände stimmen in ihrem Mittelwert von $2,02 \AA^{7}$ mit dem für das grüne $2 \mathrm{~L}-\mathrm{CsNiF}_{3}$ angegebenen Wert ${ }^{1}$ überein. Dieselben Abstände dürften wegen der Strukturverwandtschaft der genannten Verbindungen auch für das gelbe 9L- $\mathrm{CsNiF}_{3}$ zutreffen, so daß der Farbunterschied zwischen beiden $\mathrm{CsNiF}_{3}-$ Modifikationen mit Sicherheit kein Abstandseffekt ist. Eine Deutung aus den Ligandenfeldspektren im Zusammenhang mit den Strukturgegebenheiten wird im nächsten Abschnitt versucht.

\section{Ligandenfeldspektroskopische Untersuchungen des grünen und gelben $\mathrm{CsNiF}_{3}$}

Das Raumtemperaturspektrum des grünen $\mathrm{CsNiF}_{3}$ zeigt die charakteristischen drei Hauptbanden des $\mathrm{Ni}^{2+}$-Ions in oktaedrischer Koordination (Abb. 3). Die beiden deutlich verbreiterten kurzwelligen Übergänge lassen auf eine niedrigersymmetrische Ligandenfeldkomponente schließen, die bei tiefen Temperaturen zu einer Auflösung in Spaltbanden, möglicherweise überlagert von einer Spin-BahnFeinstruktur, führt. Zusätzlich ist eine markante Schwingungsfeinstruktur mit einer Progression von ca. $475 \mathrm{~cm}^{-1}$ sichtbar, die schon im $298^{\circ} \mathrm{K}$-Spektrum angedeutet war. Weiter führt die Temperaturerniedrigung zu einer deutlichen Intensitätsabnahme der Banden und verschiebt sie merklich zu größeren 
Wellenzahlen. Offenbar sind die Übergänge lediglich schwingungserlaubt, d.h. die verzerrten $\mathrm{NiF}_{6}$ Oktaeder besitzen noch immer ein Symmetriezentrum ${ }^{19}$.

Die grobe Anpassung des Tieftemperaturspektrums im Rahmen des Kristallfeldmodells ${ }^{20}$ führt zu Ligandenfeldparametern $\Delta=7900 \mathrm{~cm}^{-1}$ und $\mathrm{B}=950 \mathrm{~cm}^{-1}$ (Abb. 3). Das resultierende nephelauxetische Verhältnis $\beta=\frac{\mathrm{B}}{\mathrm{B}_{0}}=\frac{950}{1040}=10,9$ wird auch bei anderen $\mathrm{Ni}^{2+}$-Fluor-Verbindungen gefunden ${ }^{21}$. Auf eine explizite Berücksichtigung der kleinen nichtkubischen Ligandenfeldkomponente in der Kristallfeldrechnung wurde verzichtet, da die zusätzliche Schwingungsfeinstruktur in den kurzwelligen Flanken der Banden bei 13000 und $24000 \mathrm{~cm}^{-1}$ eine eindeutige Zuordnung nicht o.w. zuläßt. Der gefundene $\Delta$-Parameter liegt um $c a$.
$10 \%$ höher als die $\Delta$-Werte des gelben $\mathrm{KNiF}_{3}$ bzw. des $\mathrm{Ni}^{2+}$-dotierten $\mathrm{KMgF}_{3}{ }^{21}$. Für diese perowskitischen Verbindungen wurden Werte von $\Delta \simeq 7000 \mathrm{~cm}^{-1}$ und $\mathrm{B} \simeq 950 \mathrm{~cm}^{-1}$ ermittelt.

Die Farbe wird im wesentlichen durch das im sichtbaren Spektralbereich liegende tiefe Minimum zwischen der zweiten und dritten Hauptbande bestimmt. Aus Abb. 3 läßt sich dessen Lage zu ca. $18500 \mathrm{~cm}^{-1}(\cong 5400 \AA)$ ablesen, d.h. es liegt erwartungsgemäß im grünen Teil des sichtbaren Spektrums. Die Erniedrigung des $\Delta$-Wertes von $\mathrm{KNiF}_{3}$ gegenüber $\mathrm{CsNiF}_{3}$ um etwa $900 \mathrm{~cm}^{-1}$ bei konstantem B verschiebt die beiden kurzwelligen Banden und damit auch das zwischen ihnen liegende Minimum um mehr als $1000 \mathrm{~cm}^{-1} \mathrm{zu}$ kleineren Wellenzahlen. Dies bedingt die beobachtete gelbe Farbe von $\mathrm{KNiF}_{3}\left(17500 \mathrm{~cm}^{-1} \cong 5700 \AA\right)$.

Die diskutierten spektroskopischen Ergebnisse

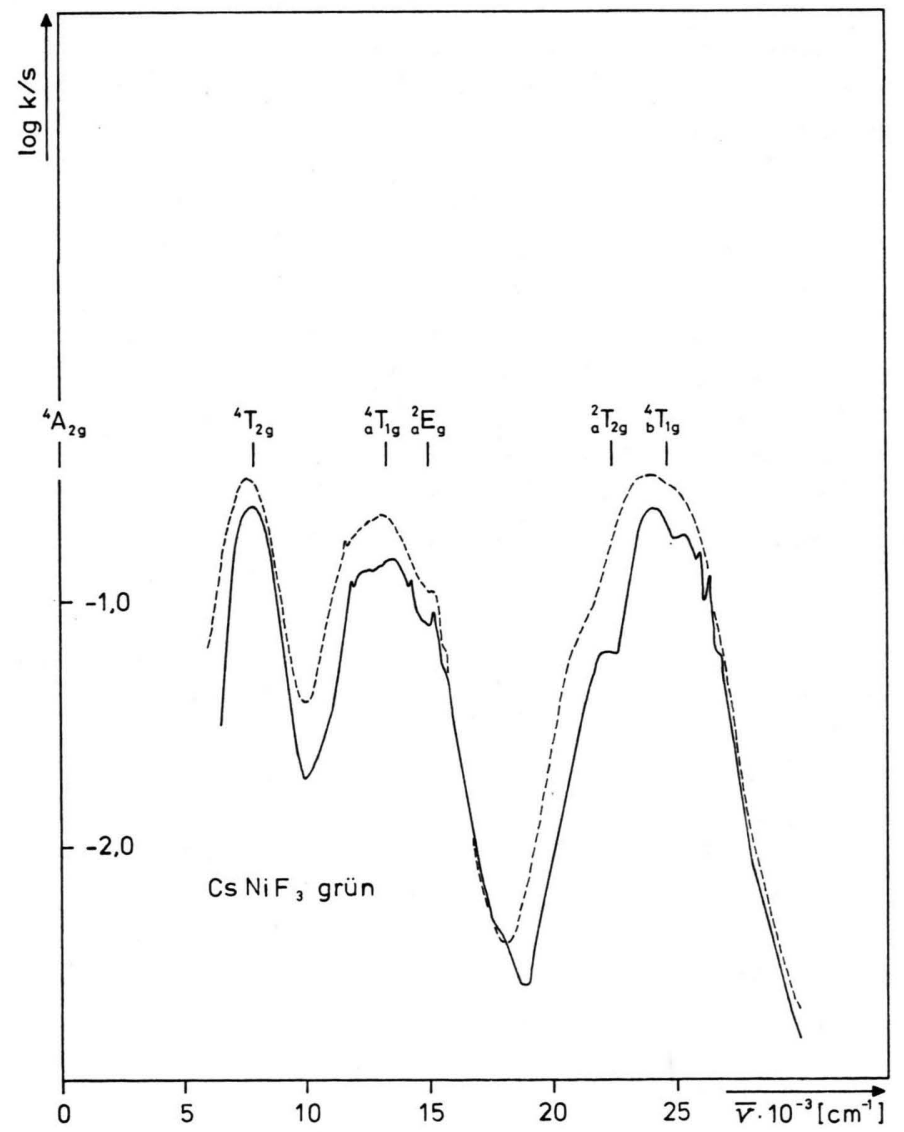

Abb. 3. Remissionsspektrum des grünen $\mathrm{CsNiF}_{3}$ (das Tieftemperaturspektrum - durchgezogene Kurve - wurde oktaedrisch mit den Parametern $\Delta=7900 \mathrm{~cm}^{-1} ; \mathrm{B}=950 \mathrm{~cm}^{-1} ; \mathrm{C} / \mathrm{B}=\mathbf{4 , 2}$ angepaßt - Punktlage mit Symmetriezentrum) 
sind in Einklang mit den bekannten Strukturen des grünen $\mathrm{CsNiF}_{3}$ sowie des $\mathrm{KNiF}_{3}$. Die flächenverknüpften $\mathrm{NiF}_{6}$-Oktaeder in der Struktur von $2 \mathrm{~L}$ $\mathrm{CsNiF}_{3}$ besitzen die Eigensymmetrie $\mathrm{D}_{3 \mathrm{~d}}$ und sind trigonal etwas gestreckt $(\mathrm{Ni}-\mathrm{F}=\mathbf{2}, 02 \AA, \Varangle \mathbf{F}-\mathrm{Ni}-\mathrm{F}=$ $\left.82,8 / 97,2^{\circ}\right)^{1}$. Die $\mathrm{Ni}^{2+}$-Ionen sind über gemeinsame $\mathrm{F}^{-}$-Ionen in leicht spitzem Winkel $\left(80,4^{\circ}\right)$ miteinander verknüpft. Die jeweils benachbarten $\mathrm{Ni}^{2+}$-Ionen können somit $\sigma$-Bindungen über zwei orthogonale $p$-Orbitale (unter Beteiligung des 2s-Orbitals) der verbrückenden Fluorliganden betätigen:

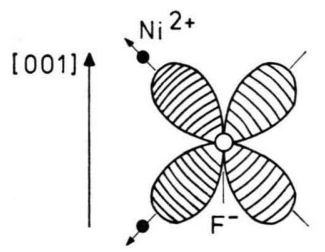

(a)

Das verbleibende senkrecht auf der $\sigma$-Bindungsebene stehende $p$-Orbital hat die für $\pi$-Bindungen zu beiden $\mathrm{Ni}^{2+}$-Nachbarn geeignete Symmetrie. $\mathrm{Da} \Delta$ die Differenz zwischen der Stärke von d-Überlappungen mit $\sigma$ - und $\pi$-Symmetrie angibt ${ }^{22}$, sollte bei zu erwartender starker $\sigma$ - und schwacher $\pi$-Bindung zwischen $\mathrm{Ni}^{2+}$ und $\mathrm{F}^{-}$ein vergleichsweise großer $\Delta$-Wert auftreten.

Im Falle einer allseitigen Eckenverknüpfung der $\mathrm{NiF}_{6}$-Oktaeder, wie sie in der kubischen Perowskitstruktur des $\mathrm{KNiF}_{3}(\mathrm{Ni}-\mathrm{F}=\mathbf{2 , 0 1} \AA)$ gegeben ist, wird die $\sigma$-Bindung zwischen zwei $\mathrm{Ni}^{2+}$ und $\mathrm{F}^{-}$ durch das gleiche $p$-Orbital (wiederum unter Beteiligung des 2 s-Orbitals) des verbindenden $\mathrm{F}^{-}$Liganden vermittelt, da die Ni-F-Ni-Geometrie linear ist:

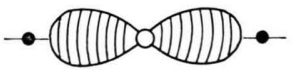

Sie sollte demzufolge, verglichen mit Fall (a), geschwächt sein (,Bindungskontrapolarisation“). Für Überlappungen mit $\pi$-Symmetrie stehen diesmal zwei $p$-Orbitale des Fluorliganden zur Verfügung, so daß stärkere $\pi$-Bindungsanteile als im grünen $\mathrm{CsNiF}_{3} \mathrm{zu}$ erwarten sind. Im Einklang mit den Beobachtungen sinkt $\Delta \mathrm{ab}$. Ähnliche Bindungseffekte in Abhängigkeit vom Kation-Ligand-KationWinkel wurden bei Chlorverbindungen dreiwertiger Übergangsmetallionen gefunden ${ }^{23}$.

Das durch gleichfalls drei Hauptbanden gekennzeichnete Raumtemperaturspektrum des gelben $\mathrm{CsNiF}_{3}$ ändert seine Intensität beim Abkühlen praktisch nicht (Abb. 4). Die Übergänge sind weniger breit als im Falle der grünen Modifikation, und im Tieftemperaturspektrum ist eine Auflösung in Spaltbanden als Folge einer niedrigersymmetrischen Ligandenfeldkomponente nur andeutungsweise sichtbar. Eine Anpassung der Bandenlagen gelingt mit den Ligandenfeldparametern $\Delta=6800 \mathrm{~cm}^{-1}$ und $\mathrm{B}=950 \mathrm{~cm}^{-1}$. Auffallend ist, da $B$ - obwohl die Hauptbanden verglichen mit der grünen Verbindung um 1000 bis $1500 \mathrm{~cm}^{-1}$ zu kleineren Energien verschoben sind - eine Schwingungsfeinstruktur im kurzwelligen Abfall der beiden energiereichen Übergänge ${ }^{3} \mathrm{~A}_{2} \rightarrow{ }_{\mathrm{a}}^{3} \mathrm{~T}_{1},{ }_{\mathrm{b}}^{3} \mathrm{~T}_{1}$ bei exakt den Wellenzahlen auftritt, bei denen eine völlig äquivalente Feinstruktur im Spektrum der grünen Modifikation beobachtet wird (Abb. 3).

Nach diesen ligandenfeldspektroskopischen Ergebnissen wird das spektrale Bild des gelben $\mathrm{CsNiF}_{3}$ dominant bestimmt durch nur schwach verzerrte oktaedrische $\mathrm{Ni}^{2+}$-Positionen ohne Symmetriezentrum: da die d-d-Übergänge nicht die für lediglich schwingungserlaubte Banden charakteristische Intensitätsabnahme mit fallender Temperatur zeigen, müssen sie Laporte-erlaubt sein. Der gegenüber der $2 \mathrm{~L}$-Struktur mit ausschließlich flächenverknüpften Oktaedern stark gesunkene $\Delta$-Wert ist dem des kubischen Perowskits $\mathrm{KNiF}_{3}$ sehr ähnlich und deutet auf lineare Fluorbrücken $\mathrm{Ni}-\mathrm{F}-\mathrm{Ni}$ hin, wie sie - zumindest partiell - in der 9L-Struktur vom $\mathrm{CsCoF}_{3}$ - bzw. $\mathrm{BaRuO}_{3}-\mathrm{Typ}^{1,8}$ vorliegen.

Zwei Drittel der Übergangsionen besetzen in diesem Strukturtyp azentrische Positionen (Eigensymmetrie $\mathrm{C}_{3 \mathrm{v}}$ ). Sie stellen die beiden endständigen Kationen in der schon erwähnten Dreiergruppe flächenverknüpfter Oktaeder dar, die über drei Fluoridionen mit den terminalen Kationen benachbarter Gruppen linear verbunden sind, während die restlichen Fluorliganden eine Flächenverknüpfung vermitteln (Abb. 1). Das letzte Drittel der Übergangsionen besetzt die zentrosymmetrische mittlere Position der Dreiergruppen (Eigensymmetrie $\mathrm{D}_{3 \mathrm{~d}}$ ). Die Nickelionen in diesen Lagen entsprechen in Symmetrie und beidseitiger Flächenverknüpfung ihrer Oktaeder denen in der 2L-Struktur. Daß sie sich im Spektrum der 9L-Form von $\mathrm{CsNiF}_{3}$ kaum bemerkbar machen, ist durch ihren schwächeren Beitrag zur Intensität als Folge des Laporte-Verbots bedingt. Die vergleichsweise $(\mathrm{Abb} .3,4)$ geringe Extinktion des Spektrums der gelben 9 L-Form hängt möglicherweise damit zusammen, daß diese 
Modifikation bei niedrigeren Temperaturen dargestellt wurde und somit als weniger gut kristallisierte Substanz geringere Korngröße besitzt. Dies wirkt sich im Reflexionsspektrum intensitätsmindernd aus. Offen bleibt die Frage, warum das gelbe $\mathrm{CsNiF}_{3}$ fast die gleichen $\Delta-$ Werte wie $\mathrm{KNiF}_{3}$ indu- ziert, obwohl die die Lichtabsorption bestimmende $\mathrm{C}_{3 \mathrm{v}}$-Position des $9 \mathrm{~L}-\mathrm{CsNiF}_{3}$ nur zur Hälfte lineare Ni-F-Ni-Verknüpfungen bildet.

Der Deutschen Forschungsgemeinschaft sowie dem Fonds der Chemischen Industrie danken wir für die Unterstützung durch Sachmittel.

Abb. 4. Remissionsspektrum des gelben $\mathrm{CsNiF}_{3}$ (das Tieftemperaturspektrum durchgezogene Kurve - wurde oktaedrisch mit den Parametern

$\Delta=6800 \mathrm{~cm}^{-1} ; \mathrm{B}=950 \mathrm{~cm}^{-1} ; \mathrm{C} / \mathrm{B}=4,2$ angepaßt - Punktlage ohne Symmetriezentrum).

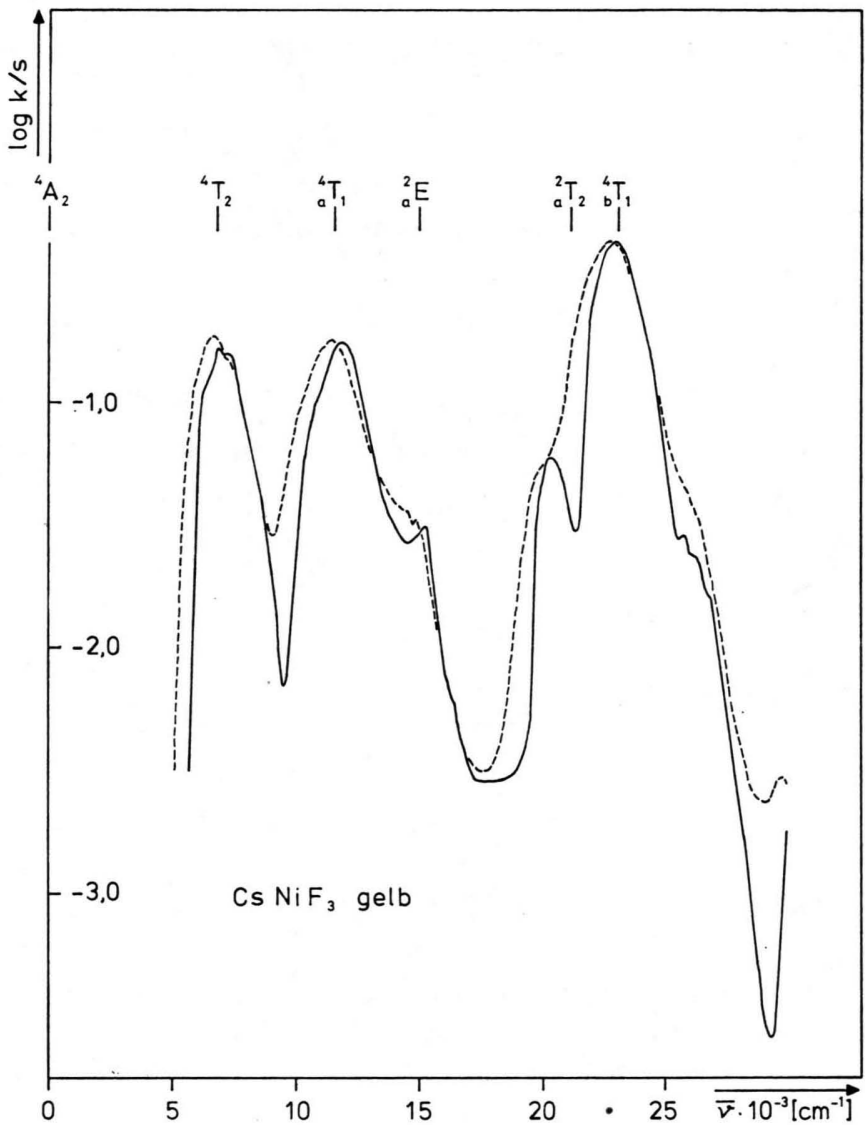

1 D. Babel, Z. Anorg. Allg. Chem. 369, 117 [1969].

2 G. N. Tischenko, Trudy Inst. Kristallogr. Akad. Nauk SSSR 11, 93 [1955].

3 J. J. Lander, Acta Crystallogr. 4, 148 [1951].

4 D. BABEL, Z. Naturforsch. 20 a, 165 [1965].

5 M. Steiner, W. Krüger und D. Babel, Solid State Commun. 9, 227 [1971].

6 M. Steiner, H. Dachs und D. Babel, AIP Conference Proceedings "Magnetism and Magnetic Materials - 1972", 10, 664 [1973].

7 D. BABEL, Vortrag auf dem 2. Europäischen Fluorsymposium, Göttingen 1968.

8 P. C. Donohue, L. Katz und R. WARD, Inorg. Chem. 4, 306 [1965].

9 J. M. Longo und J. A. Kafalas, J. Appl. Phys. 40, 1601 [1969].

10 J. M. Longo und J. A. Kafalas, J. Solid State Chem. 1, 103 [1969].

11 R. Haegele, Dissertation, Marburg 1974.

12 H. Bode und E. Voss, Z. Anorg. Allg. Chem. 286, 136 [1956].
13 D. Babel, Z. Anorg. Allg. Chem. 387, 161 [1972].

14 D. Babel, G. Pausewang und W. Viebahn, Z. Naturforsch. 22b, 1219 [1967].

15 R. Jesse und R. Hoppe, Z. Anorg. Allg. Chem. 403, 143 [1974].

16 D. Reinen, C. Friebel und V. Propach, Z. Anorg. Allg. Chem. 408, 187 [1974].

17 J. Arndt, D. Babel, R. Haegele und N. Rombach, Z. Anorg. Allg. Chem. [1975], im Druck.

18 D. BABEL, Structure and Bonding 3, 1 [1967].

19 J. Grefer und D. Reinen, Z. Anorg. Allg. Chem. 404, 167 [1974].

20 D. Rernen, Ber. Bunsenges. Phys. Chem. 69, 82 [1965].

21 J. Ferguson, D. L. Wood and K. Knox, J. Chem. Phys. 39, 881 [1963].

22 z.B.: D. Reinen, Angew. Chem. 24, 991 [1971]; Int. Ed. 10, 901 [1971].

23 P. Köhler und D. ReInen, in Vorbereitung. 